\title{
Variants of Lipid-Related Genes in Adult Japanese Patients with Severe Hypertriglyceridemia
}

\author{
Akira Matsunaga ${ }^{1}$, Mariko Nagashima ${ }^{1}$, Hideko Yamagishi ${ }^{1}$ and Keijiro Saku ${ }^{2}$ \\ ${ }^{1}$ Department of Laboratory Medicine, Fukuoka University School of Medicine, Fukuoka, Japan \\ ${ }^{2}$ Department of Cardiology, Fukuoka University School of Medicine, Fukuoka, Japan
}

Aim: Hypertriglyceridemia is a type of dyslipidemia that contributes to atherosclerosis and coronary heart disease. Variants in lipoprotein lipase $(L P L)$, apolipoprotein CII (APOC2), apolipoprotein AV (APOA5), glycosylphosphatidylinositol-anchored high-density lipoprotein-binding protein 1 (GPIHBP1), lipase maturation factor $1(L M F 1)$, and glucokinase regulator $(G C K R)$ are responsible for hypertriglyceridemia. We investigated the molecular basis of severe hypertriglyceridemia in adult patients referred to the Clinical Laboratory at Fukuoka University Hospital.

Methods: Twenty-three adult patients with severe hypertriglyceridemia $(>1,000 \mathrm{mg} / \mathrm{dL}, 11.29 \mathrm{mmol} / \mathrm{L})$ were selected. The coding regions of candidate genes were sequenced by next-generation sequencing. Forty-nine genes reportedly associated with hypertriglyceridemia were analyzed.

Results: In the 23 patients, we detected 70 variants: 28 rare and 42 common ones. Among the 28 rare variants with $<1 \%$ allele frequency, p.I4533L in $A P O B$, p.M490I in $M L X I P L$, p.L152M in NCAN, and p.S264T in TIMD4 were novel. We did not observe single gene homozygous or compound heterozygous disease-causing rare variants in any of the 23 hypertriglyceridemia cases. However, in silico algorithms and previous reports indicated that five rare variants, APOA5 (p.T184S), GCKR (c.354+1G>A), LMF1 (p.G410R), and LRP1 (p.G813R; p.R2173Q), and seven common variants, $A P O A 5$ (pG185C), $A P O E$ (p.C130R; p.E262K/p.E263K), GCKR (p.V103M), GPIHBP1 (p.C14F), LRP1 (p.Y4054F), and MLXIPL (p.Q241H), can cause hypertriglyceridemia. However, all five disease-causing rare variants detected in this study were heterozygous.

Conclusions: The prevalence of disease-causing rare variants in candidate genes in severe hypertriglyceridemia patients was low. The major causes of severe hypertriglyceridemia were not single gene abnormalities, but involved multiple gene variations and environmental factors.

See editorial vol. 27: 1255-1256

Key words: Severe hypertriglyceridemia, Chylomicronemia, Variant, Mutation, Next-generation sequencing

\section{Introduction}

Numerous clinical outcome studies have shown that the levels of circulating triglyceride (TG) are an independent risk factor for coronary artery disease in humans ${ }^{1}$. Severe hypertriglyceridemia is a common lipid disorder that is associated with many comorbidities, including acute pancreatitis ${ }^{2}$. Furthermore, the presence of severe hypertriglyceridemia correlates with a series of secondary factors, including extreme obesity, uncontrolled diabetes, severe liver/renal insuffi- ciency, thyroid disease, and chemotherapy. Plasma TG is largely contained in TG-rich lipoproteins, such as very low-density lipoprotein (VLDL) and chylomicron particles. Lipoprotein lipase (LPL) is a critical enzyme in determining plasma triglyceride levels and LPL variants can result in severe hypertriglyceridemia. Moreover, apoprotein (apo)A-V, apoC-II, glycosylphosphatidylinositol-anchored high-density lipoprotein binding protein 1 (GPIHBP1), and lipase maturation factor 1 (LMF1) are also co-factors involved in the activation, transportation, or matura-

Address for correspondence: Akira Matsunaga, Department of Laboratory Medicine, Fukuoka University School of Medicine, 7-45-1 Nanakuma, Johnan-ku, Fukuoka 814-0180, Japan E-mail: matsunag@cis.fukuoka-u.ac.jp

Received: June 28, 2019 Accepted for publication: January 8, 2020

Copyright@2020 Japan Atherosclerosis Society

This article is distributed under the terms of the latest version of CC BY-NC-SA defined by the Creative Commons Attribution License. 
tion of LPL. Defects in LPL, APOA5, APOC2, GPI$H B P 1$, and $L M F 1$ are closely associated with chylomicronemia ${ }^{3,4)}$. In addition, tribbles homolog1 (TRIB1), carbohydrate response element-binding protein [ChREBP (MLXIPL)], and glucokinase regulator (GCKR) are all expressed in the liver and contribute to hepatic lipogenesis ${ }^{5)}$. TRIB1 upregulates lipogenic gene expression via the indirect regulation of $\mathrm{C} / \mathrm{EBP} \alpha$, while ChREBP directly binds to lipogenic gene promoters. When glycogen stores are full, GCKR regulates the production of glucose-6-phosphate, a substrate for lipogenesis. Hepatic lipogenesis yields triglyceride to growing nascent VLDL particles, which are subsequently secreted from the liver into the circulation.

Common variations at more than 200 genomic loci are associated at genome-wide levels of significance with one or more plasma lipid traits. However, many of the identified genes have no recognized role in lipoprotein metabolism, indicating that an incredible wealth of novel lipid biology remains to be discovered.

The majority of cases of severe hypertriglyceridemia are diagnosed in adulthood and the molecular basis of the condition has not been fully defined. There may be ethnic differences in the causative variants of hypertriglyceridemia, although this is poorly understood. In this study, we investigated the prevalence and characteristics of serum triglyceride-related gene variants in adult Japanese patients with severe hypertriglyceridemia. Forty-nine genes reported to be associated with hypertriglyceridemia, including $L P L$, $A P O C 2$, GPIHBP1, APOA5, and LMF1, were analyzed by next-generation sequencing (NGS) ${ }^{3,6-8)}$.

\section{Materials and Methods}

\section{Sample Selection}

This single-center study was conducted at Fukuoka University Hospital between August 2017 and October 2018. The subjects were patients who visited because of severe hypertriglyceridemia or patients in whom high triglyceride levels were detected by the hospital clinical laboratory. After an overnight fast, blood samples were collected. Twenty-three patients aged over 20 years with severe hypertriglyceridemia [TG $>1,000 \mathrm{mg} / \mathrm{dL}(11.29 \mathrm{mmol} / \mathrm{L})]$ were included. Average daily alcohol intake (grams of ethanol per day) was calculated from the clinical records. The subjects were divided into four subgroups according to ethanol consumption per day (nondrinkers; light drinkers: $<10 \mathrm{~g}$ of ethanol per day; moderate drinkers: $\geq 10 \mathrm{~g}$ and $<30 \mathrm{~g}$ of ethanol per day; heavy drinkers: $\geq 30 \mathrm{~g}$ of ethanol per day). Written informed consent was obtained from each patient prior to DNA analysis. This study was approved by the ethics committee of Fukuoka University (\#2017M009).

\section{Whole-Exome Sequencing}

Genomic DNA was extracted from blood samples using a SepaGene kit (Sekisui Medical, Tokyo, Japan), in accordance with the manufacturer's instructions. Exome enrichment was carried out with $3 \mu \mathrm{g}$ of genomic DNA using an Agilent SureSelect Human All Exon V6 Kit (Agilent Technologies, Santa Clara, $\mathrm{CA}$ ), in accordance with the manufacturer's protocol. Each sample was sequenced to at least $100 \times$ raw target depth, by the $100 \mathrm{bp}$ paired-end sequencing method on the Illumina HiSeq 2000 platform (Illumina, San Diego, CA).

We checked several parameters of the fastq files, including base quality score distribution, sequence quality score distribution, average base content per read, GC distribution in the reads, PCR amplification issue, over-represented sequences, and adapter trimming. Sequence reads were trimmed where necessary and low-quality sequence reads were excluded to retain only high-quality sequences for further analysis. Adapter trimming was performed using Trimmomatic-0.36 (http://www.usadellab.org/cms/index. php?page=trimmomatic). Paired-end reads were aligned to the reference human genome primary assembly hg19 downloaded from the University of California Santa Cruz Genome Browser Database. Alignment was performed using BWA (version bwa0.7.12). Paired reads that mapped to two different chromosomes were discarded from the analysis. Aligned reads were first sorted by Picard tools, and read duplicates were removed using the Picard Mark Duplicates command. After removing the duplicates, reads were realigned around the known indels provided by the Genome Analysis Toolkit (GATK) group. This was followed by a base recalibration step. After recalibration, the quality score of each base was more accurate. Known variant positions were taken into account to recalibrate the quality score. After realignment, we used GATK v3.4-46 to identify singlenucleotide variants (SNVs) and short indels. We further filtered the variants to retain good quality (depth and variant score) ones. Identified variants were annotated using Annovar (http://annovar.openbioinformatics.org/en/latest/user-guide/download/). To evaluate the pathogenicity of the identified nonsynonymous variants, we used SIFT (https://sift.bii.a-star.edu.sg), PolyPhen-2 (http://genetics.bwh.harvard.edu/pph2/), Mutation Taster (http://www.mutationtaster.org), and FATHMM (http://fathmm.biocompute.org.uk) programs to predict the functional consequences of the 
observed amino acid substitutions. ClinVar (https:// www.ncbi.nlm.nih.gov/clinvar/) was also screened for variant evaluation. Splicing variants, variants pointed out by multiple in silico predictions and not excluded by ClinVar, and variants previously reported as the cause of hypertriglyceridemia were defined as diseasecausing variants, 9-14). The Exome Aggregation Consortium Database (ExAC) contains allele frequency data for over 60,000 individuals, including American, Latino, East Asian, Finnish, Non-Finnish European, and South Asian (http://exac.broadinstitute.org) individuals. The Human Genetic Variation Database (HGVD) contains allele frequency data for 1208 Japanese individuals (http://www.hgvd.genome.med. kyoto-u.ac.jp). We searched for variants with an allele frequency of less than $10 \%$ in ExAC. However, for variants that had already been reported to be associated with hypertriglyceridemia, we also searched for variants with a high allele frequency ${ }^{6,9,15,16)}$. Rare and common variants were defined as variants present at a frequency of $<1 \%$ and $>1 \%$ of those obtained from ExAC and HGVD, respectively. Since HGVD presents the Japanese allele frequency, the variants with $<$ $1 \%$ allele frequency in both ExAC and HGVD were defined as rare variants. The variants with $>1 \%$ allele frequency in ExAC or HGVD were defined as common variants. The bam files of variants detected in NGS sequences were visually confirmed using Integrative Genomics Viewer (IGV) (http://software.broadinstitute.org/software/igv/home).

The following 49 genes that have been reported to be related to hypertriglyceridemia were analyzed: AKR1C4, ANGPTL3, ANGPTL4, ANGPTL8, APOA1, APOA5, APOB, APOC2, APOC3, APOE, BLK, CETP, CILP2, CREB3L3, FADS1, FADS2, FADS3, FTO, GALNT2, GCKR, GPD1, GPIHBP1, INSR, IRS1, LDLR, LIPC, LMF1, LPL, LRPAP1, LRP1, MIR148A, MET, MLXIPL, MPP3, NAT2, NCAN, NDST1, NROB2, PEPD, PIGV, PDXDC1, PINX1, PLTP, PPARA, SEL1L, TIMD4, TRIB1, $V E G F A$, and $X K R 6^{6,9,15-25)}$.

\section{Plasma Lipid and Lipoprotein Analysis}

Total plasma cholesterol, TG, high-density-lipoprotein cholesterol (HDL-C), and low-density-lipoprotein cholesterol (LDL-C) levels were determined by enzymatic methods using reagents from Sekisui Medical (Tokyo, Japan). Plasma apoA-I, A-II, B, C-II, $\mathrm{C}$-III, and $\mathrm{E}$ were measured using a turbidimetric immunoassay method from Sekisui Medical (Tokyo, Japan).

\section{Results}

Among the 23 patients with severe hypertriglyceridemia, 16 were male, 11 were obese (body mass index >25), 17 consumed alcohol (7 light, 2 moderate, and 8 heavy drinkers), 10 had diabetes, 1 had pancreatitis, and 5 had fatty liver (Table 1). Fourteen patients were taking lipid-lowering drugs. Table 2 shows plasma lipid and apolipoprotein levels at the maximum triglyceride level and the time of blood biopsy. The mean maximum triglyceride level for all 23 patients was $2107 \mathrm{mg} / \mathrm{dL}$. As shown in Table 2, statins were given in four cases (No. 3, 6, 10, 15), but only one case (No. 23) showed LDL-C levels above $140 \mathrm{mg} / \mathrm{dL}$. ApoB, CII, CIII, and E levels were elevated in 17 of 23 cases (No. 2, 4-13, 15-18, 21, 23).

In a whole-exome analysis of 49 genes, 70 variants were detected in 31 genes (Tables 3 and 4). Among them, there were 45 variants with $<1 \%$ allele frequency in ExAC, and 28 rare variants with $<1 \%$ allele frequency in both ExAC and HGVD (Japanese) (Table 4). There were 42 common variants. Of these, 17 variants had $<1 \%$ ExAC allele frequency and $>$ $1 \%$ HGVD allele frequency (Japanese).

As shown in Tables 3 and 4, in 18 of the 23 patients (No. 1-5, 7, 9-12, 14, 16-19, 21-23), the following 28 rare variants were found: $A N G P T L 3$ (p.Y104H, No. 11), APOA5 (p.T184S, No. 4, 14), $A P O B$ (p.R1388H, No. 10; p.D2065G, No. 7; p. I2950T, No.12; p.N2964S, No. 12; p.K3232E, No. 22; p.I4533L, No. 3), CILP2 (p.A579G, No. 10; p. R1142W, No. 2), FTO (p.D144N, No. 24), GALNT2 (p.G40S, No. 5), GCKR (c.354+1G>A, No. 21), INSR (p.Y1361C, No. 19), IRS1 (p.M209T, No. 21), LMF1 (p.M159V, No. 4; p.G410R, No. 17, 21), LRP1 (p.G813R, No. 10; p.R2173Q, No. 1), MET (p.L211W, No. 3; p.M636V, No. 16), MLXIPL (p.M490I, No. 18), NCAN (p.L152M, No. 22), NROB2 (p.R216H, No. 3), PDXDC1 (p.R142Q, No. 9), TIMD4 (p.E216K, No. 14, 22; p.S264T, No. 17), and XKR6 (p.A103S, No. 18). Among them, four variants, p.I4533L in $A P O B$ (No. 3), p.M490I in MLXIPL (No. 18), p.L152M in NCAN (No. 22), and p.S264T in TIMD4 (No. 17), were novel. As shown in Table 4, we obtained four function prediction scores using PolyPhen-2, SIFT, MutationTaster, and FATHMM.

As shown in Tables 1, 3, and 4, in five patients (No. 6, 8, 13, 15, 20) only the following common variants were observed: ANGPTL4 (p.T266M), $A P O A 5$ (p.V153M; p.G185C), APOB (p.12_15del; p.R532W; p.N2785H), APOE (p.C130R; p.R176C), GCKR (p.L446P), GPIHBP1 (p.C14F), LIPC (p.V95M), LMF1 (p.P562R), and NCAN (p.P92S; 
Table 1. Clinical characteristics and disease-causing variants of 23 patients with severe hypertriglyceridemia

\begin{tabular}{|c|c|c|c|c|c|c|c|c|c|c|c|c|c|}
\hline \multirow{2}{*}{ No. } & \multirow{2}{*}{$\begin{array}{l}\text { Age } \\
\text { (yr) }\end{array}$} & \multirow{2}{*}{ Sex } & \multirow{2}{*}{$\begin{array}{l}\text { TG Max } \\
(\mathrm{mg} / \mathrm{dL})\end{array}$} & \multirow[t]{2}{*}{ BMI } & \multirow{2}{*}{ DM } & \multirow{2}{*}{$\begin{array}{l}\text { Drink- } \\
\text { ing }\end{array}$} & \multirow{2}{*}{$\begin{array}{l}\text { Pancre- } \\
\text { atitis }\end{array}$} & \multirow{2}{*}{$\begin{array}{l}\text { Fatty } \\
\text { Liver }\end{array}$} & \multirow{2}{*}{$\begin{array}{l}\text { Lipid-lowering } \\
\text { Therapy }\end{array}$} & \multicolumn{2}{|c|}{ Number of Variants } & \multicolumn{2}{|c|}{ Disease-causing Variants ${ }^{\mathrm{s}}$} \\
\hline & & & & & & & & & & Rare & Common & Rare & Common \\
\hline 1 & 22 & M & 1159 & 32.3 & + & non & - & - & Bezafibrate $400 \mathrm{mg}$ & 1 & 9 & LRP1 (p.R2173Q) & $\begin{array}{l}\text { APOA5 (p.G185C), } \\
\text { MLXIPL (p.Q241H) }\end{array}$ \\
\hline 2 & 41 & M & 1553 & 26.3 & + & light & - & - & No & 1 & 7 & & $A P O E$ (p.C130R) \\
\hline 3 & 54 & M & 2133 & 24.5 & - & $\begin{array}{l}\text { moder- } \\
\text { ate }\end{array}$ & - & - & $\begin{array}{l}\text { EPA 1,800 mg, } \\
\text { Rosuvastatin } 5 \mathrm{mg}\end{array}$ & 3 & 11 & & $\begin{array}{l}\text { APOA5 (p.G185C), } \\
\text { GCKR (p.V103M) }\end{array}$ \\
\hline 4 & 47 & M & 1077 & 36.7 & - & heavy & - & + & No & 2 & 11 & APOA5 (p.T184S) & $A P O E$ (p.C130R) \\
\hline 5 & 53 & $\mathrm{~F}$ & 1130 & 19.0 & - & heavy & - & - & Fenofibrate $53.3 \mathrm{mg}$ & 1 & 6 & & GPIHBP1 (p.C14F*) \\
\hline 6 & 38 & M & 1503 & 31.5 & + & heavy & - & - & $\begin{array}{l}\text { EPA/DHA } 2.0 \mathrm{~g}, \\
\text { Rosuvastatin } 2.5 \mathrm{mg}\end{array}$ & 0 & 6 & & \\
\hline 7 & 20 & $\mathrm{~F}$ & 1555 & 26.2 & + & non & - & + & No & 1 & 8 & & $\begin{array}{l}A P O E \\
\text { (p.E262K/p.E263K) }\end{array}$ \\
\hline 8 & 44 & $\mathrm{~F}$ & 2675 & 16.7 & - & light & - & + & Fenofibrate $53.3 \mathrm{mg}$ & 0 & 6 & & MLXIPL (p.Q241H) \\
\hline 9 & 45 & M & 1955 & 23.7 & - & light & - & - & Fenofibrate $160 \mathrm{mg}$ & 1 & 11 & & $\begin{array}{l}A P O A 5 \text { (p.G185C), } \\
\text { GPIHBPI (p.C14F), } \\
A P O E \text { (p.C130R) }\end{array}$ \\
\hline 10 & 46 & M & 1543 & 31.1 & - & heavy & - & + & Pitavastatin $1 \mathrm{mg}$ & 3 & 8 & LRP1 (p.G813R) & GPIHBP1 (p.C14F) \\
\hline 11 & 56 & $\mathrm{~F}$ & 1879 & 23.6 & + & heavy & - & + & No & 1 & 6 & & $\begin{array}{l}L R P 1 \text { (p.Y4054F), } \\
M L X I P L(\text { p.Q241H), } \\
\text { APOE (p.C130R) }\end{array}$ \\
\hline 12 & 57 & $\mathrm{~F}$ & 2437 & 27.7 & + & non & - & - & No & 2 & 7 & & GPIHBP1 (p.C14F) \\
\hline 13 & 49 & M & 1174 & 25.9 & - & light & - & - & EPA $1800 \mathrm{mg}$ & 0 & 8 & & $\begin{array}{l}\text { APOA5 (p.G185C), } \\
\text { GPIHBP1 (p.C14F) }\end{array}$ \\
\hline 14 & 58 & M & 1208 & 24.1 & + & non & - & - & EPA $1800 \mathrm{mg}$ & 2 & 6 & APOA5 (p.T184S) & GPIHBP1 (p.C14F) \\
\hline 15 & 58 & M & 2135 & 22.6 & + & heavy & - & - & Rosuvastatin $2.5 \mathrm{mg}$ & 0 & 7 & & GPIHBP1 (p.C14F) \\
\hline 16 & 55 & $\mathrm{~F}$ & 3307 & 19.1 & - & non & - & - & Fenofibrate $80 \mathrm{mg}$ & 1 & 12 & & $\begin{array}{l}\text { APOA5 (p.G185C*), } \\
\text { GPIHBPI (p.C14F), } \\
\text { LRP1 (p.Y4054F), } \\
\text { APOE (p.C130R*) }\end{array}$ \\
\hline 17 & 48 & M & 1083 & 25.2 & - & light & - & - & No & 2 & 4 & $L M F 1$ (p.G410R) & $\begin{array}{l}\text { GKCR (p.V103M), } \\
\text { GPIHBP1 (p.C14F) }\end{array}$ \\
\hline 18 & 43 & M & 2545 & 29.0 & + & light & - & - & Fenofibrate $53.3 \mathrm{mg}$ & 2 & 11 & & $\begin{array}{l}\text { GPIHBP1 (p.C14F), } \\
A P O E \text { (p.C130R) }\end{array}$ \\
\hline 19 & 35 & $\mathrm{~F}$ & 2517 & 20.1 & - & light & - & - & $\begin{array}{l}\text { EPA/DHA } 4.0 \mathrm{~g}, \\
\text { Fenofibrate } 80 \mathrm{mg}\end{array}$ & 1 & 9 & & GPIHBP1 (p.C14F) \\
\hline 20 & 23 & M & 2767 & 23.3 & - & heavy & + & - & No & 0 & 8 & & $\begin{array}{l}\text { MLXIPL (p.Q241H), } \\
A P O E \text { (p.C130R) }\end{array}$ \\
\hline 21 & 39 & M & 6089 & 35.7 & - & non & - & - & No & 3 & 8 & $\begin{array}{l}\text { GCKR (c.354+1G>A), } \\
L M F 1 \text { (p.G410R) }\end{array}$ & APOE (p.C130R) \\
\hline 22 & 23 & M & 2459 & 24.6 & - & heavy & - & - & No & 3 & 7 & & $\begin{array}{l}\text { GPIHBP1 (p.C14F*), } \\
\text { MLXIPL (p.Q241H) }\end{array}$ \\
\hline
\end{tabular}


(Cont. Table 1)

\begin{tabular}{|c|c|c|c|c|c|c|c|c|c|c|c|c|c|}
\hline \multirow[t]{2}{*}{ No. } & \multirow{2}{*}{$\begin{array}{l}\text { Age } \\
(\mathrm{yr})\end{array}$} & \multirow[t]{2}{*}{ Sex } & \multirow{2}{*}{$\begin{array}{l}\text { TG Max } \\
(\mathrm{mg} / \mathrm{dL})\end{array}$} & \multirow[t]{2}{*}{ BMI } & \multirow[t]{2}{*}{ DM } & \multirow{2}{*}{\multicolumn{2}{|c|}{$\begin{array}{l}\text { Drink- Pancre- } \\
\text { ing atitis }\end{array}$}} & \multirow{2}{*}{$\begin{array}{l}\text { Fatty } \\
\text { Liver }\end{array}$} & \multirow{2}{*}{$\begin{array}{l}\text { Lipid-lowering } \\
\text { Therapy }\end{array}$} & \multicolumn{2}{|c|}{ Number of Variants } & \multicolumn{2}{|c|}{ Disease-causing Variants $\$$} \\
\hline & & & & & & & & & & Rare & Common & Rare & Common \\
\hline 23 & 43 & M & 2349 & 23.6 & + & $\begin{array}{l}\text { moder- } \\
\text { ate }\end{array}$ & - & - & $\begin{array}{l}\text { EPA/DHA } 4.0 \mathrm{~g}, \\
\text { Bezafibrate } 400 \mathrm{mg}\end{array}$ & 1 & 10 & & $\begin{array}{l}A P O A 5 \text { (p.G185C), } \\
\text { GPIHBP1 (p.C14F), } \\
A P O E \text { (p.C130R) }\end{array}$ \\
\hline
\end{tabular}

yr, years old; M, male; F, female; TG Max, maximum triglyceride value

BMI, body mass index; DM, diabetes mellitus

EPA, eicosapentaenoic acid; DHA, docosahexaenoic acid

non, nondrinkers; light, light drinkers: $<10 \mathrm{~g}$ of ethanol per day; moderate, moderate drinkers: $\geq 10 \mathrm{~g}$ and $<30 \mathrm{~g}$ of ethanol per day; heavy, heavy drinkers: $\geq 30 \mathrm{~g}$ of ethanol per day

Rare, rare variants with $<1 \%$ allele frequency in both ExAC and HGVD; Common, common variants with $>1 \%$ allele frequency in ExAC or HGVD; ${ }^{*}$, homozygote

$\$$, Disease-causing variants were defined as splicing variants, variants that were identified by multiple in silico predictions and not excluded by ClinVar, and variants that were previously reported as a cause of hypertriglyceridemia.

APO, APOLIPOPROTEIN; GCKR, GLUCOKINASE REGULATORY PROTEIN; GPIHBP1, GLYCOSYLPHOSPHATIDYLINOSITOLANCHORED HIGH-DENSITY LIPOPROTEIN-BINDING PROTEIN 1; LMF1, LIPASE MATURATION FACTOR 1; LRP1, LOW-DENSITY LIPOPROTEIN RECEPTOR-RELATED PROTEIN 1; MLXIPL, MLX-INTERACTING PROTEIN-LIKE

\section{p.S838N).}

No nonsynonymous variants were found in 18 genes: APOC2, APOC3, BLK, FADS1, FADS2, FADS3, GPD1, LDLR, LRPAP1, MIR148A, MPP3, NDST1, PEPD, PIGV, PLTP, SEL1L, TRIB1, and VEGFA.

We detected five rare variants [APOA5 (p.T184S), GCKR (c.354+1G>A), LMF1 (p.G410R), LRP1 (p.R2173Q; p.G813)] and seven common variants [APOA5 (p.G185C), APOE (p.C130R; p.E262K/p.E263K), GCKR (p.V103M), GPIHBP1 (p.C14F), LRP1 (p.Y4054F), MLXIPL (p.Q241H)] that could be disease-causing (Tables 1, 3 , and 4). However, we did not observe cases of hypertriglyceridemia caused by single gene homozygosity or compound heterozygosity of disease-causing rare variants.

\section{Discussion}

We searched for variants in lipid-related genes in severe adult hypertriglyceridemia patients. Severe hypertriglyceridemia is divided into two distinct primary forms. One of these, familial chylomicronemia, is a very rare monogenic early-onset chylomicronemia, which presents in childhood or adolescence. Most of these cases involve type 1 hyperlipidemia. The other form, polygenic late-onset chylomicronemia, is caused by an accumulation of several genetic variants and can be exacerbated by secondary factors, such as poor diet, obesity, alcohol intake, and diabetes mellitus. Most of these cases involve type 5 hyperlipidemia with elevated VLDL and chylomicron particles. Many genetic studies of familial chylomicronemia have been reported, most of which excluded cases with obesity, diabetes, and alcohol consumption and focused on single gene abnormalities $5,26,27$. However, the majority of patients with severe hypertriglyceridemia encountered in the clinic have at least one of these risk factors. Therefore, in this study, we did not exclude cases with such risk factors and analyzed a large number of lipid-related genes. In this study, plasma levels of apoB were elevated in 17 of 23 cases. The proportion of plasma apoB48 present in chylomicron particles is very low; therefore, elevated apoB levels indicate that most cases involved type 5 hyperlipidemia with elevated VLDL and chylomicron particles.

Severe hypertriglyceridemia, usually resulting from a combination of genetic and environmental factors, may increase the risk of acute pancreatitis. In pancreatitis resulting from severe hypertriglyceridemia, triglyceride levels were reported to be more than $1,500 \mathrm{mg} / \mathrm{dL}(16.935 \mathrm{mmol} / \mathrm{L})^{28)}$. In this study, the maximum triglyceride level was over $1,500 \mathrm{mg} / \mathrm{dL}$ in 17 cases but pancreatitis was associated with only one case.

We analyzed LPL, GPIHBP1, APOA5, APOC2, and $L M F 1$, which all cause familial chylomicronemia. We detected no variants in $A P O C 2$. In $L P L$, only p. S474X heterozygotes as a gain-of-function variant were observed in three cases (Table 3) ${ }^{3,29)}$. A common variant of GPIHBP1 (p.C14F) was found in 13 patients (No. 5, 9, 10, 12-19, 22, 23) and was homozygous in three of these. In silico algorithms indicated that this variant could be benign (Table 4). However, GPIHBP1 (p.C14F) shows normal LPL binding activity, but the level of the mutant protein was reduced in vitro $^{30,31)}$. Therefore, p.C14F may be a cause of hyper- 
Table 2. Serum lipid and apolipoprotein levels for 23 patients with severe hypertriglyceridemia

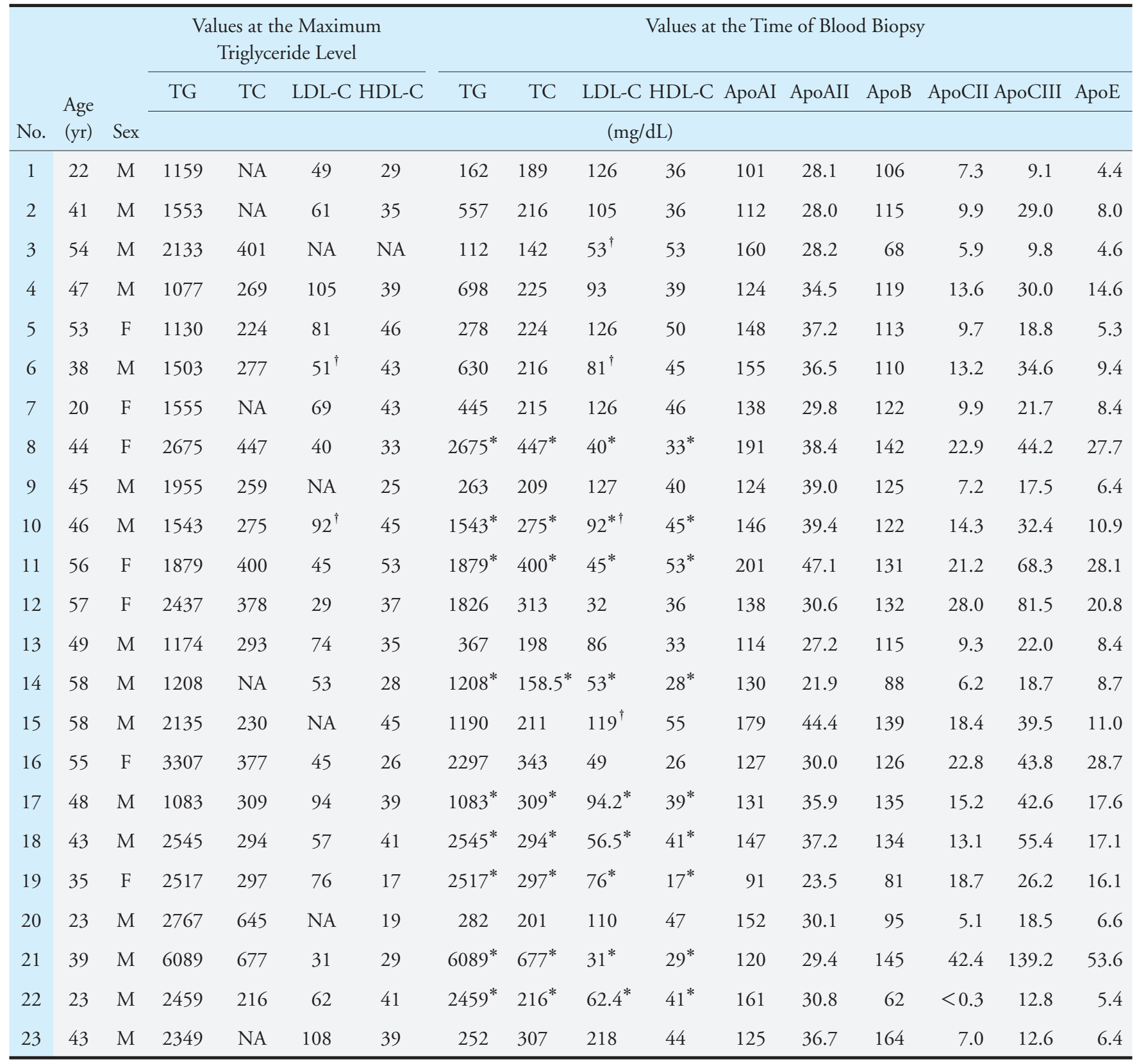

yr, years old; $M$, male; $F$, female

NA, not available; ${ }^{*}$, same values as maximum triglyceride level; ${ }^{\dagger}$, statins were given in four cases

triglyceridemia. Among the 23 patients, 16 were heterozygous for $A P O A 5$ variants. A disease-causing rare heterozygous variant, p.T184S, was found in two patients (No. 4, 14). A disease-causing common variant, p.G185C, was detected in six patients (No. 1, 3, 9, 13, 16, 23) ${ }^{32-34)}$. Variants in $L M F 1$ were found in eight patients. One patient (No. 4) had two heterozygous variants, one rare (p.M159V) and one common (p.P562R). p.G410R was heterozygous in two patients (No. 17, 21) and was predicted to be disease-causing by in silico analysis ${ }^{11)}$.

$A P O E$ variants affect triglyceride levels ${ }^{9,10)}$ and were observed in 10 patients (Table 3). Among disease-causing common variants, p.C130R was found in eight cases. Another disease-causing common variant, p.E262K, p.E263K, was heterozygous in one case (No. 7). LIPC encodes hepatic triglyceride lipase. A common variant of $L I P C$, p.V95M, was found in 14 cases including one homozygote; however, p.V95M is common in the Japanese population, as shown in Table 4, 
Table 3. Genetic variants of the main candidate genes in 23 patients with severe hypertriglyceridemia

\begin{tabular}{|c|c|c|c|c|c|c|c|c|c|c|}
\hline No. & $\begin{array}{l}\text { Age } \\
\text { (yr) }\end{array}$ & Sex & $\begin{array}{l}\text { TG Max } \\
\text { (mg/dL) }\end{array}$ & $\begin{array}{c}\text { APOE } \\
\text { Phenotype }\end{array}$ & ANGPTL3 & ANGPTL4 & APOA5 & APOB & APOE & CILP2 \\
\hline 1 & 22 & M & 1159 & $3 / 3$ & - & - & p.G185C $\$$ & $\begin{array}{l}\text { p.12_15del, } \\
\text { p.N2785H }\end{array}$ & - & - \\
\hline 2 & 41 & M & 1553 & $3 / 4$ & - & - & - & - & p.C130R $\$$ & p.R1142W \\
\hline 3 & 54 & M & 2133 & $2 / 3$ & - & p.T266M & p.G185C & $\begin{array}{l}\text { p.12_15del*, } \\
\text { p.I4533L" }\end{array}$ & p.R176C & - \\
\hline 4 & 47 & M & 1077 & $3 / 4$ & - & - & p.T184S & p.R4270T & p.C130R & - \\
\hline 5 & 53 & $\mathrm{~F}$ & 1130 & $3 / 3$ & - & p.T266M & p.V153M & - & - & - \\
\hline 6 & 38 & M & 1503 & $3 / 3$ & - & - & p.V153M & p.N2785H & - & - \\
\hline 7 & 20 & F & 1555 & $3 / 7$ & - & p.V123M & - & $\begin{array}{l}\text { p.12_15del*, } \\
\text { p.D2065G }{ }^{*}\end{array}$ & $\begin{array}{l}\text { p.E262K/ } \\
\text { p.E263K }\end{array}$ & - \\
\hline 8 & 44 & F & 2675 & $3 / 3$ & - & - & p.V153M & - & - & - \\
\hline 9 & 45 & M & 1955 & $3 / 4$ & - & - & $\begin{array}{l}\text { p.V153M, } \\
\text { p.G185C }\end{array}$ & - & p.C130R & - \\
\hline 10 & 46 & M & 1543 & $3 / 3$ & - & - & - & $\begin{array}{l}\text { p.D550H, } \\
\text { p.R1388H }\end{array}$ & - & p.A579G \\
\hline 11 & 56 & $\mathrm{~F}$ & 1879 & $3 / 4$ & p.Y104H & p.T266M & - & - & p.C130R & - \\
\hline 12 & 57 & $\mathrm{~F}$ & 2437 & $3 / 3$ & - & p.T266M & p.V153M & $\begin{array}{l}\text { p.I2950T } T^{\#} \\
\text { p.N2964S }\end{array}$ & - & - \\
\hline 13 & 49 & M & 1174 & $3 / 3$ & - & p.T266M & p.G185C $\$$ & - & - & - \\
\hline 14 & 58 & M & 1208 & $3 / 3$ & - & - & p.T184S & $\begin{array}{l}\text { p.R532W, } \\
\text { p.N2785H }\end{array}$ & - & - \\
\hline 15 & 58 & M & 2135 & $3 / 3$ & - & - & p.V153M & - & - & - \\
\hline 16 & 55 & $\mathrm{~F}$ & 3307 & $4 / 4$ & - & p.V123M & p.G185C $* \$$ & $\begin{array}{l}\text { p.D550H, } \\
\text { p.E3788K }\end{array}$ & p.C130R*\$ & - \\
\hline 17 & 48 & M & 1083 & $3 / 3$ & - & p.T266M & - & - & - & - \\
\hline 18 & 43 & M & 2545 & $3 / 4$ & - & - & p.V153M & p.R532W & p.C130R & - \\
\hline 19 & 35 & $\mathrm{~F}$ & 2517 & $3 / 3$ & - & - & - & p.R532W & - & - \\
\hline 20 & 23 & M & 2767 & $3 / 4$ & - & - & - & p.12_15del & p.C130R & - \\
\hline 21 & 39 & M & 6089 & $3 / 4$ & - & - & p.V153M & p.T194M & p.C130R & - \\
\hline 22 & 23 & M & 2459 & $3 / 3$ & - & - & p.V153M & $\begin{array}{l}\text { p.T194M, } \\
\text { p.K3232E }\end{array}$ & - & - \\
\hline 23 & 43 & $\mathrm{M}$ & 2349 & $3 / 4$ & - & p.T266M & $\begin{array}{l}\text { p.V153M, } \\
\text { p.G185C }\end{array}$ & p.R532W* & p.C130R & - \\
\hline
\end{tabular}


(Cont Table 3)

\begin{tabular}{|c|c|c|c|c|c|c|c|c|}
\hline No. & GCKR & GPIHBP1 & LIPC & LMF1 & LPL & LRP1 & MLXIPL & TIMD4 \\
\hline 1 & - & - & - & p.P562R & p.S474X & p.R2173Q ${ }^{\#} \$$ & $\begin{array}{l}\text { p.Q241H } \\
\text { p.A358V }\end{array}$ & - \\
\hline 2 & - & - & p.V95M & - & p.S474X & - & - & - \\
\hline 3 & $\begin{array}{l}\text { p.V103M }{ }^{\$}, \\
\text { p.L446P* }\end{array}$ & - & p.V95M & p.P562R & - & - & - & - \\
\hline 4 & p.L446P & - & p.V95M & $\begin{array}{l}\text { p.M159V } \\
\text { p.P562R }\end{array}$ & p.S474X & - & - & - \\
\hline 5 & - & p.C14F*\$ & - & - & - & - & - & - \\
\hline 6 & p.L446P & - & p.V95M & - & - & - & - & - \\
\hline 7 & - & - & p.V95M & - & - & - & - & - \\
\hline 8 & p.L446P & - & p.V95M & - & - & - & $\begin{array}{l}\text { p.Q241H } \\
\text { p.A358V }\end{array}$ & - \\
\hline 9 & p.L446P & p.C14F & p.V95M & p.P562R & - & - & - & - \\
\hline 10 & p.L446P & p.C14F ${ }^{* \$}$ & p.V95M & - & - & p.G813R & - & - \\
\hline 11 & p.L446P* & - & - & - & - & p.Y4054F & $\begin{array}{l}\text { p.Q241H } \\
\text { p.A358V }\end{array}$ & - \\
\hline 12 & - & p.C14F & - & p.P562R & - & - & - & - \\
\hline 13 & p.L446P & p.C14F & p.V95M & p.P562R & - & - & - & - \\
\hline 14 & - & p.C14F & - & - & - & - & - & p.E216K \\
\hline 15 & p.L446P & p.C14F & - & - & - & - & - & - \\
\hline 16 & p.L446P & p.C14F & - & - & - & p.Y4054F & - & - \\
\hline 17 & $\begin{array}{l}\text { p.V103M }{ }^{\$}, \\
\text { p.L446P* }\end{array}$ & p.C14 $\mathrm{F}^{\$}$ & - & p.G410R ${ }^{\# \$}$ & - & - & - & p.S264T \\
\hline 18 & - & p.C14F & p.V95M & - & - & - & p.M490I & - \\
\hline 19 & p.L446P* & p.C14F & p.V95M & - & - & - & - & - \\
\hline 20 & p.L446P & - & p.V95M* & - & - & - & $\begin{array}{l}\text { p.Q241H }{ }^{\$}, \\
\text { p.A358V }\end{array}$ & - \\
\hline 21 & $\begin{array}{l}\text { c. } 354+1 G>A^{\# \$} \\
\text { p. } L 446 P^{*}\end{array}$ & - & p.V95M & p.G410R ${ }^{\# \$}$ & - & - & - & - \\
\hline 22 & p.L446P & p.C14F*\$ & - & - & - & - & $\begin{array}{l}\text { p.Q241H } \\
\text { p.A358V }\end{array}$ & p.E216K \\
\hline 23 & p.L446P* & p.C14F & p.V95M & - & - & - & - & - \\
\hline
\end{tabular}

yr, years old; M, male; F, female; TG Max, maximum triglyceride value

*, homozygote; ${ }^{\#}$, rare variants with $<1 \%$ allele frequency in both ExAC and HGVD; ${ }^{\$}$, disease-causing variants (Disease-causing variants were defined as splicing variants, variants that were identified by multiple in silico predictions and not excluded by ClinVar, and variants that were previously reported as a cause of hypertriglyceridemia.)

ANGPTL, ANGIOPOIETIN-LIKE; APO, APOLIPOPROTEIN; CILP2, CARTILAGE INTERMEDIATE LAYER PROTEIN 2; GCKR, GLUCOKINASE REGULATORY PROTEIN; GPIHBP1, GLYCOSYLPHOSPHATIDYLINOSITOL-ANCHORED HIGH DENSITY LIPOPROTEIN-BINDING PROTEIN 1; LIPC, HEPATIC LIPASE; LMF1, LIPASE MATURATION FACTOR 1; LPL, LIPOPROTEIN LIPASE; LRP1, LOW DENSITY LIPOPROTEIN RECEPTOR-RELATED PROTEIN 1; MLXIPL, MLX-INTERACTING PROTEIN-LIKE; NCAN, NEUROCAN CORE PROTEIN PRECURSOR; TIMD4, T-CELL IMMUNOGLOBULIN AND MUCIN DOMAINS-CONTAINING PROTEIN 4 
Table 4. Genetic variants identified in candidate genes in 23 patients with severe hypertriglyceridemia

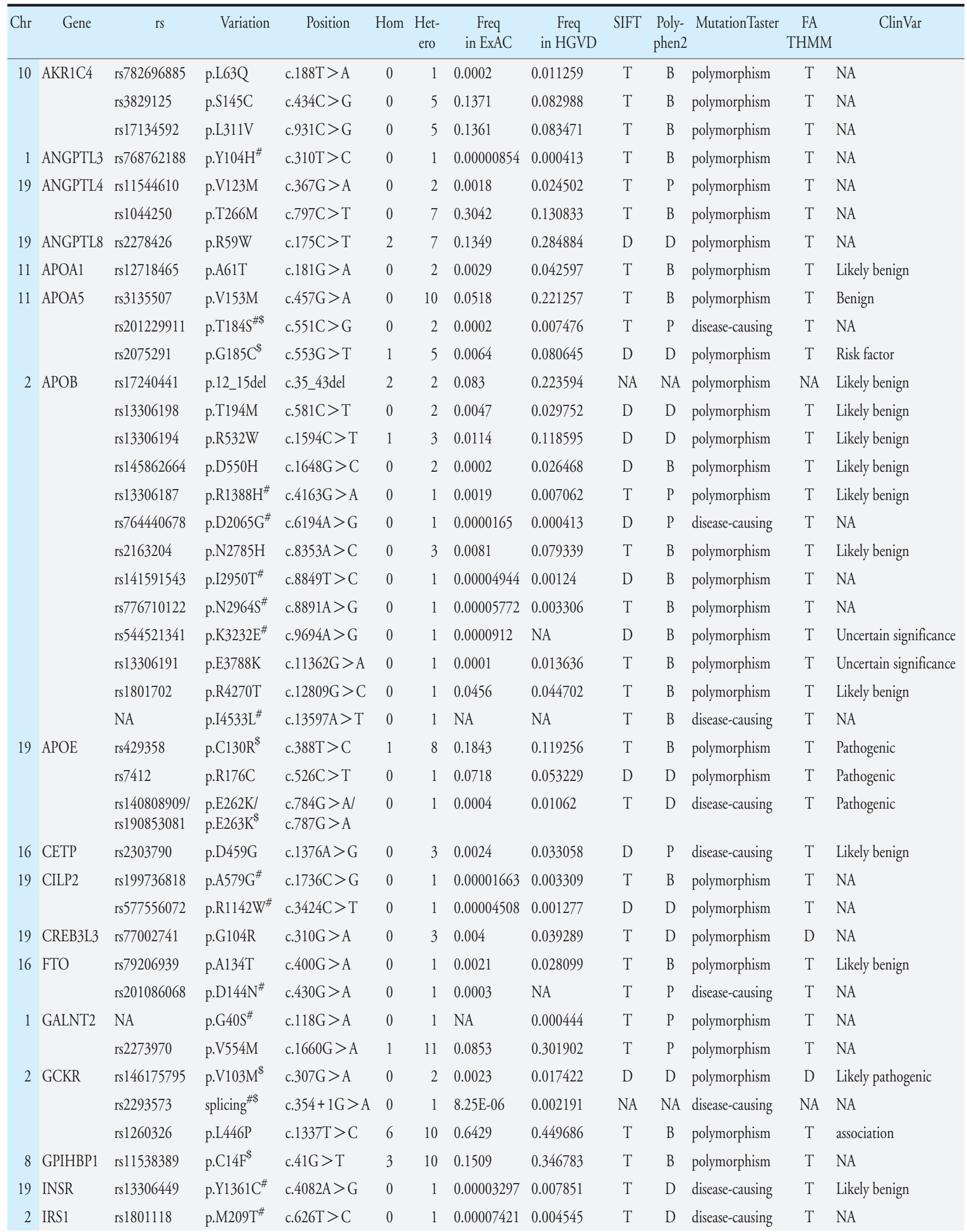


(Cont Table 4)

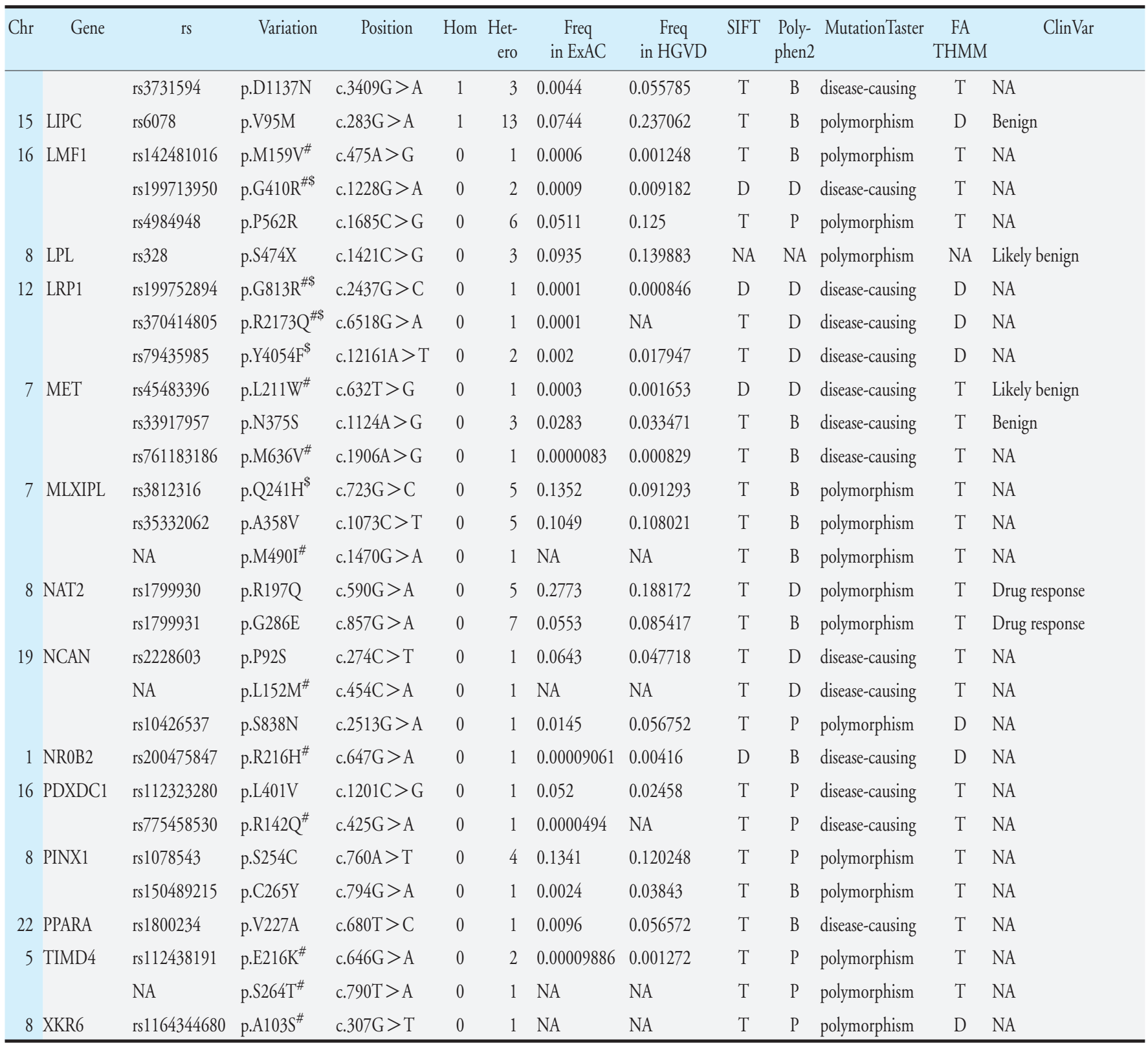

Chr, chromosome; rs, reference SNP ID number

Freq in ExAC, Frequency in Exome Aggregation Consortium Database (World)

Freq in HGVD, Frequency in Human Genetic Variation Database (Japanese)

Hom, number of homozygotes; Het, number of heterozygotes; NA, not available

SIFT prediction: T, Tolerated; D, Deleterious; Polyphen2, Polymorphism Phenotyping v2: B, Benign; P. Possibly Damaging; D, Probably Damaging FATHMM, Functional Analysis through Hidden Markov Models: T, Tolerated; D, Deleterious

\#, rare variants with $<1 \%$ allele frequency in both ExAC and HGVD; $\$$, disease-causing variants (Disease-causing variants were defined as splicing variants, variants that were identified by multiple in silico predictions and not excluded by ClinVar, and variants that were previously reported as a cause of hypertriglyceridemia.)

AKR1C4, ALDO-KETO REDUCTASE FAMILY 1, MEMBER C4; ANGPTL, ANGIOPOIETIN-LIKE; APO, APOLIPOPROTEIN; CETP, CHOLESTERYL ESTER TRANSFER PROTEIN; CILP2, CARTILAGE INTERMEDIATE LAYER PROTEIN 2; CREB3L3, cAMP RESPONSE ELEMENT-BINDING PROTEIN 3-LIKE 3; FTO, FAT MASS- AND OBESITY-ASSOCIATED GENE; GALNT2, GaINAc TRANSFERASE 2; GCKR, GLUCOKINASE REGULATORY PROTEIN; GPIHBP1, GLYCOSYLPHOSPHATIDYLINOSITOL-ANCHORED HIGH DENSITY LIPOPROTEIN-BINDING PROTEIN 1; INSR, INSULIN RECEPTOR; IRS1,INSULIN RECEPTOR SUBSTRATE 1; LIPC, HEPATIC LIPASE; LMF1, LIPASE MATURATION FACTOR 1; LPL, LIPOPROTEIN LIPASE; LRP1, LOW DENSITY LIPOPROTEIN RECEPTORRELATED PROTEIN 1; MET, MET PROTOONCOGENE;MLXIPL, MLX-INTERACTING PROTEIN-LIKE; NAT2, N-ACETYLTRANSFERASE 2; NCAN, NEUROCAN CORE PROTEIN PRECURSOR; NR0B2, NUCLEAR RECEPTOR SUBFAMILY 0, GROUP B, MEMBER 2; PDXDC1,PYRIDOXAL-DEPENDENT DECARBOXYLASE DOMAIN-CONTAINING PROTEIN 1; PINX1, PIN2-INTERACTING PROTEIN 1; PPARA, PEROXISOME PROLIFERATOR-ACTIVATED RECEPTOR-ALPHA; TIMD4, T-CELL IMMUNOGLOBULIN AND MUCIN DOMAINS-CONTAINING PROTEIN 4; XKR6, XK-RELATED PROTEIN 6 
and is not associated with hypertriglyceridemia ${ }^{17)}$.

All three angiopoietin-like protein (ANGPTL) members, ANGPTL3, ANGPTL4, and ANGPTL8, are LPL inhibitors and deficiency or overexpression of any one of them results in hypotriglyceridemia or hypertriglyceridemia, respectively ${ }^{18)}$. In $A N G P T L 3$, p.Y104H was heterozygous in one patient (No. 11). The ANGPTL4 variant was detected in nine cases (No. $3,5,7,11-13,16,17,23)$. In the ANGPTL8 gene, p.R59W was detected in nine cases. However, we could not detect disease-causing variants in the $A N G$ PTL3, 4, and 8 genes $^{35)}$.

$A P O B$ encodes apolipoprotein $\mathrm{B}$, which is found in all atherogenic lipoprotein species. Various $A P O B$ variants were detected in 15 of the 23 cases in this study (No. 1, 3, 4, 6, 7, 10, 12, 14, 16, 18-23). A novel variant, p.I4533L, was heterozygous in one case (No. 3). p.12_15del is associated with higher levels of apoB and LDL cholesterol without affecting triglyceride levels ${ }^{36,37)}$. p.N2785H has no effect on hyperlipidemia risk in the Chinese population ${ }^{37)}$. No diseasecausing variants were detected in the apo $\mathrm{B}$ gene. The glucokinase regulatory protein encoded by $G C K R$ is an allosteric regulator that allows rapid mobilization of glucokinase in response to glucose concentration. For $G C K R$, three variants were observed in 16 patients. It was reported that p.P446L promoted glucose uptake and mobilization, leading to increased de novo triglyceride synthesis, whereas p.L446P had the opposite effects ${ }^{38)}$. The heterozygous common variant p.V103M and the rare splicing donor site variant c.354+1G >A (rs2293573) were detected in two cases (No. 3, 17) and one case (No. 21), respectively. As shown in Table 4, p.V103M was predicted to be disease-causing by in silico algorithms (SIFT, PolyPhen-2, and FATHMM). The low-density-lipoprotein receptor-related protein 1 (LRP1) is a member of the low-density-lipoprotein receptor (LDLR) family ${ }^{39)}$. LRP1 was originally identified as an endocytic receptor for $\alpha 2$-macroglobulin-proteinase complexes and apolipoprotein E. LRP1 is involved in the clearance of chylomicron remnants from circulation. LRP1 functions in signal transduction pathways and can interact with other cell receptors. LRP1 is also involved in insulin signaling and glucose homeostasis in several different tissues. For $L R P 1$, two rare variants, p.G813R and p.R2173Q, and the common variant p.Y4054F were heterozygous in one (No. 10), one (No. 1), and two cases (No. 11, 16), respectively. In silico algorithms (PolyPhen-2, Mutation Taster, and FATHMM) indicated that these three variants could be diseasecausing (Table 4). MLXIPL encodes a transcription factor that regulates glucose utilization and lipogenesis in the liver. MLXIPL is associated with plasma triglyc- eride levels in the Japanese population ${ }^{9)}$. In $M L X I P L$, a novel mutant, p.M490I, was heterozygous in one case. A disease-causing common variant, p.Q241H, was heterozygous in five cases (No. 1, 8, 11, 20, 22) ${ }^{14)}$. A common variant, p.A358V, was also reported to be associated with apoA1 values in Chinese subjects ${ }^{40)}$. The Neurocan-Cartilage intermediate layer protein 2 (NCAN-CILP2) region spans $300 \mathrm{~kb}$ on chromosome 19 and forms a tight linkage disequilibrium block. Through GWAS, many genetic variants associated with plasma lipid levels have been identified, among which the NCAN-CILP2 region has high statistical significance and a relatively large effect size per allele on LDL cholesterol and triglyceride levels ${ }^{19)}$. Two rare heterozygous variants, p.A579G (No. 10) and p. R1142W (No. 2), were found in the CILP2 gene. For the NCAN gene, a novel variant p.L152M (No. 22) was heterozygous. However, no disease-causing variants were detected in the NCAN and CILP2 genes. The T-cell immunoglobulin and mucin domain 4 (TIMD4) gene is located on chromosome 5 and is a member of the T-cell immunoglobulin domain and mucin domain gene family, which plays a critical role in regulating immune responses. TIMD4 variants are associated with serum lipid traits, but the findings regarding their effects have been inconsistent ${ }^{41)}$. For TIMD4, a novel variant, p.S264T, and a rare variant, p.E216K, were heterozygous in one (No. 17) and two cases (No. 14, 22), respectively. The function of XKrelated protein 6 (XKRG) has been poorly defined ${ }^{20)}$. A rare variant of the $X K R 6$ gene, p.A103S, was heterozygous in one patient (No. 18). No disease-causing variants were detected in the TIMD 4 and XKR6 genes.

Until recently, most genetic studies on patients with severe hypertriglyceridemia focused on $L P L$ and genes that regulate LPL, namely, $A P O A 5, A P O C 2$, GPHLBP1, and LMF1; other genetic causes may thus have been neglected ${ }^{32,42,43)}$. In the current study, we performed NGS for 49 reported TG-regulating genes in 23 Japanese patients with severe hypertriglyceridemia. In this study, there were 17 variants with frequency $<1 \%$ in ExAC for the global population, but $>1 \%$ in HGVD for the Japanese. This appears to indicate the existence of ethnic differences in allele frequency.

Most of our cases were believed to involve type 5 hyperlipidemia. Surendran et al. analyzed five genes (LPL, GPIHBP1, APOA5, APOC2, and LMF1) in patients with severe hypertriglyceridemia ${ }^{3)}$. In 29 of 43 cases $(67 \%)$ of type 1 hyperlipidemia and 11 of 43 cases $(26 \%)$ of type 5 hyperlipidemia, rare variants causing disease were detected. In 9 cases $(21 \%)$ of type 1 hyperlipidemia and 16 cases (37\%) of type 5 hyperlipidemia, common variants of LPL or APOA5 were 
also detected ${ }^{3)}$. Although the target genes and ethnicity of the subjects in the current study were different, here, five disease-causing rare variants in four genes, APOA5 (p.T184S), GCKR (c.354+1G>A), LMF1 (p.G410R), and LRP1 (p.G813R; p.R2173Q), were detected in 6 out of 23 cases (26\%). In 16 cases (70\%), only 7 disease-causing common variants in six genes, APOA5 (pG185C), APOE (p.C130R; p. E262K/p.E263K), GCKR (p.V103M), GPIHBP1 (p.C14F), LRP1 (p.Y4054F), and MLXIPL (p.Q241H), were detected. In one case (4\%), no disease-causing variants were detected (Table 1). However, we have to recognize that severe hypertriglyceridemia could involve a gene that has yet to be characterized. These cases highlight the need for physicians to consider a polygenic cause for patients with severe hypertriglyceridemia. This possibility should be evaluated using methods that screen for both rare largeeffect variants and common small-effect variants. We detected several homozygotes or compound heterozygotes of disease-causing common variants, but all of the disease-causing rare variants detected in this study were heterozygotes.

This study had some limitations. First, we could not prepare a normal control group. Second, this was a single-center study and the sample size was small. Third, only exome sequencing was performed. However, to the best of our knowledge, few studies have performed detailed analysis of more than 40 genes in cases of severe hypertriglyceridemia.

\section{Conclusion}

The prevalence of rare variants in candidate genes in patients with severe hypertriglyceridemia was low. The major causes of severe hypertriglyceridemia were not single gene variants, but multiple genetic variations and environmental factors.

\section{Conflict of Interest} declare.

The authors have no conflicts of interest to

\section{Acknowledgements}

This work was supported by a Grant-in-Aid for Scientific Research (JP16K08955).

\section{References}

1) Wilson PW and Grundy SM: The metabolic syndrome: a practical guide to origins and treatment: Part II. Circulation, 2003; 108: 1537-1540
2) Murphy MJ, Sheng X, MacDonald TM and Wei L: Hypertriglyceridemia and acute pancreatitis. JAMA Intern Med, 2013; 173: 162-164

3) Surendran RP, Visser ME, Heemelaar S, Wang J, Peter J, Defesche JC, Kuivenhoven JA, Hosseini M, Peterfy M, Kastelein JJ, Johansen CT, Hegele RA, Stroes ES and Dallinga-Thie GM: Mutations in LPL, APOC2, APOA5, GPIHBP1 and LMF1 in patients with severe hypertriglyceridaemia. J Intern Med, 2012; 272: 185-196

4) Chokshi N, Blumenschein SD, Ahmad Z and Garg A: Genotype-phenotype relationships in patients with type I hyperlipoproteinemia. J Clin Lipidol, 2014; 8: 287-295

5) Caddeo A, Mancina RM, Pirazzi C, Russo C, Sasidharan K, Sandstedt J, Maurotti S, Montalcini T, Pujia A, Leren TP, Romeo $S$ and Pingitore P: Molecular analysis of three known and one novel LPL variants in patients with type I hyperlipoproteinemia. Nutr Metab Cardiovasc Dis, 2018; 28: 158-164

6) Bauer RC, Khetarpal SA, Hand NJ and Rader DJ: Therapeutic Targets of Triglyceride Metabolism as Informed by Human Genetics. Trends Mol Med, 2016; 22: 328-340

7) Chen WJ, Sun XF, Zhang RX, Xu MJ, Dou TH, Zhang XB, Zhong M, Yang WQ, Liu L, Lu XY and Zhu CQ: Hypertriglyceridemic acute pancreatitis in emergency department: Typical clinical features and genetic variants. J Dig Dis, 2017; 18: 359-368

8) Jin JL, Sun D, Cao YX, Zhang HW, Guo YL, Wu NQ, Zhu CG, Gao Y, Dong QT, Liu G, Dong Q and Li JJ: Intensive genetic analysis for Chinese patients with very high triglyceride levels: Relations of mutations to triglyceride levels and acute pancreatitis. EBioMedicine, 2018; 38: 171-177

9) Nakayama K, Bayasgalan T, Yamanaka K, Kumada M, Gotoh T, Utsumi N, Yanagisawa Y, Okayama M, Kajii E, Ishibashi S, Iwamoto $S$ and Jichi Community Genetics T: Large scale replication analysis of loci associated with lipid concentrations in a Japanese population. J Med Genet, 2009; 46: 370-374

10) Matsunaga A, Sasaki J, Moriyama K, Arakawa F, Takada Y, Nishi K, Hidaka K and Arakawa K: Population frequency of apolipoprotein E5 (Glu3-->Lys) and E7 (Glu244-$>$ Lys, Glu245-->Lys) variants in western Japan. Clin Genet, 1995; 48: 93-99

11) Lee CJ, Oum CY, Lee Y, Park S, Kang SM, Choi D, Jang Y, Lee JH and Lee SH: Variants of Lipolysis-Related Genes in Korean Patients with Very High Triglycerides. Yonsei Med J, 2018; 59: 148-153

12) Shetty S, Xing C and Garg A: Type 1 Hyperlipoproteinemia Due to Compound Heterozygous Rare Variants in GCKR. J Clin Endocrinol Metab, 2016; 101: 3884-3887

13) Xie SL, Chen TZ, Huang XL, Chen C, Jin R, Huang ZM and Zhou MT: Genetic Variants Associated with Gestational Hypertriglyceridemia and Pancreatitis. PLoS One, 2015; 10: e0129488

14) Kooner JS, Chambers JC, Aguilar-Salinas CA, Hinds DA, Hyde CL, Warnes GR, Gomez Perez FJ, Frazer KA, Elliott P, Scott J, Milos PM, Cox DR and Thompson JF: Genome-wide scan identifies variation in MLXIPL associated with plasma triglycerides. Nat Genet, 2008; 40: 149 151

15) Cefalu AB, Spina R, Noto D, Ingrassia V, Valenti V, 
Giammanco A, Fayer F, Misiano G, Cocorullo G, Scrimali C, Palesano O, Altieri GI, Ganci A, Barbagallo CM and Averna MR: Identification of a novel LMF1 nonsense mutation responsible for severe hypertriglyceridemia by targeted next-generation sequencing. J Clin Lipidol, 2017; 11: 272-281 e278

16) Lewis GF, Xiao C and Hegele RA: Hypertriglyceridemia in the genomic era: a new paradigm. Endocr Rev, 2015; 36: 131-147

17) van Hoek M, Dallinga-Thie GM, Steyerberg EW and Sijbrands EJ: Diagnostic value of post-heparin lipase testing in detecting common genetic variants in the LPL and LIPC genes. Eur J Hum Genet, 2009; 17: 1386-1393

18) Zhang R: The ANGPTL3-4-8 model, a molecular mechanism for triglyceride trafficking. Open Biol, 2016; 6: 150272

19) Boonvisut S, Nakayama K, Makishima S, Watanabe K, Miyashita H, Lkhagvasuren M, Kagawa Y and Iwamoto S: Replication analysis of genetic association of the NCANCILP2 region with plasma lipid levels and non-alcoholic fatty liver disease in Asian and Pacific ethnic groups. Lipids Health Dis, 2016; 15: 8

20) Johansen CT, Wang J, Lanktree MB, Cao H, McIntyre AD, Ban MR, Martins RA, Kennedy BA, Hassell RG, Visser ME, Schwartz SM, Voight BF, Elosua R, Salomaa V, O'Donnell CJ, Dallinga-Thie GM, Anand SS, Yusuf S, Huff MW, Kathiresan S and Hegele RA: Excess of rare variants in genes identified by genome-wide association study of hypertriglyceridemia. Nat Genet, 2010; 42: 684687

21) Lamiquiz-Moneo I, Blanco-Torrecilla C, Bea AM, MateoGallego R, Perez-Calahorra S, Baila-Rueda L, Cenarro A, Civeira $\mathrm{F}$ and de Castro-Oros I: Frequency of rare mutations and common genetic variations in severe hypertriglyceridemia in the general population of Spain. Lipids Health Dis, 2016; 15: 82

22) Stahel P, Xiao C, Hegele RA and Lewis GF: Polygenic Risk for Hypertriglyceridemia Can Mimic a Major Monogenic Mutation. Ann Intern Med, 2017; 167: 360-361

23) Lillis AP, Van Duyn LB, Murphy-Ullrich JE and Strickland DK: LDL receptor-related protein 1: unique tissuespecific functions revealed by selective gene knockout studies. Physiol Rev, 2008; 88: 887-918

24) Hegele RA, Ban MR, Cao H, McIntyre AD, Robinson JF and Wang J: Targeted next-generation sequencing in monogenic dyslipidemias. Curr Opin Lipidol, 2015; 26: 103-113

25) He PP, Jiang T, OuYang XP, Liang YQ, Zou JQ, Wang Y, Shen QQ, Liao L and Zheng XL: Lipoprotein lipase: Biosynthesis, regulatory factors, and its role in atherosclerosis and other diseases. Clin Chim Acta, 2018; 480: 126-137

26) Khovidhunkit W, Charoen S, Kiateprungvej A, Chartyingcharoen P, Muanpetch S and Plengpanich W: Rare and common variants in LPL and APOA5 in Thai subjects with severe hypertriglyceridemia: A resequencing approach. J Clin Lipidol, 2016; 10: 505-511 e501

27) Martin-Campos JM, Julve J, Roig R, Martinez S, Errico TL, Martinez-Couselo S, Escola-Gil JC, Mendez-Gonzalez J and Blanco-Vaca F: Molecular analysis of chylomicronemia in a clinical laboratory setting: diagnosis of 13 cases of lipoprotein lipase deficiency. Clin Chim Acta,
2014; 429: 61-68

28) Rashid N, Sharma PP, Scott RD, Lin KJ and Toth PP: Severe hypertriglyceridemia and factors associated with acute pancreatitis in an integrated health care system. J Clin Lipidol, 2016; 10: 880-890

29) Zhang H, Henderson H, Gagne SE, Clee SM, Miao L, Liu G and Hayden MR: Common sequence variants of lipoprotein lipase: standardized studies of in vitro expression and catalytic function. Biochim Biophys Acta, 1996; 1302: 159-166

30) Yamamoto $H$, Onishi M, Miyamoto N, Oki R, Ueda H, Ishigami M, Hiraoka $\mathrm{H}$, Matsuzawa $\mathrm{Y}$ and Kihara S: Novel combined GPIHBP1 mutations in a patient with hypertriglyceridemia associated with CAD. J Atheroscler Thromb, 2013; 20: 777-784

31) Charriere S, Peretti N, Bernard S, Di Filippo M, Sassolas A, Merlin M, Delay M, Debard C, Lefai E, Lachaux A, Moulin P and Marcais C: GPIHBP1 C89F neomutation and hydrophobic C-terminal domain G175R mutation in two pedigrees with severe hyperchylomicronemia. J Clin Endocrinol Metab, 2011; 96: E1675-1679

32) Pullinger CR, Aouizerat BE, Movsesyan I, Durlach V, Sijbrands EJ, Nakajima K, Poon A, Dallinga-Thie GM, Hattori H, Green LL, Kwok PY, Havel RJ, Frost PH, Malloy MJ and Kane JP: An apolipoprotein A-V gene SNP is associated with marked hypertriglyceridemia among Asian-American patients. J Lipid Res, 2008; 49: 1846-1854

33) Matsunaga A, Arishima $H$, Niimura $H$, Zhang B, Uehara Y, Ohwaki K, Morita M, Hayashida K and Saku K: Strong linkage disequilibrium and association of $-1131 \mathrm{~T}>\mathrm{C}$ and c.553G $>\mathrm{T}$ polymorphisms of the apolipoprotein A5 gene with hypertriglyceridemia in a Japanese population. Circ J, 2007; 71: 746-752

34) Evans D, Aberle J and Beil FU: Resequencing the apolipoprotein A5 (APOA5) gene in patients with various forms of hypertriglyceridemia. Atherosclerosis, 2011; 219: 715-720

35) Quagliarini F, Wang Y, Kozlitina J, Grishin NV, Hyde R, Boerwinkle E, Valenzuela DM, Murphy AJ, Cohen JC and Hobbs HH: Atypical angiopoietin-like protein that regulates ANGPTL3. Proc Natl Acad Sci U S A, 2012; 109: 19751-19756

36) Niu C, Luo Z, Yu L, Yang Y, Chen Y, Luo X, Lai F and Song Y: Associations of the APOB rs693 and rs17240441 polymorphisms with plasma APOB and lipid levels: a meta-analysis. Lipids Health Dis, 2017; 16: 166

37) Gu QL, Han Y, Lan YM, Li Y, Kou W, Zhou YS, Hai XJ, Yan $\mathrm{B}$ and $\mathrm{Ci} \mathrm{CH}$ : Association between polymorphisms in the APOB gene and hyperlipidemia in the Chinese Yugur population. Braz J Med Biol Res, 2017; 50: e6613

38) Beer NL, Tribble ND, McCulloch LJ, Roos C, Johnson PR, Orho-Melander M and Gloyn AL: The P446L variant in GCKR associated with fasting plasma glucose and triglyceride levels exerts its effect through increased glucokinase activity in liver. Hum Mol Genet, 2009; 18: 40814088

39) Au DT, Strickland DK and Muratoglu SC: The LDL Receptor-Related Protein 1: At the Crossroads of Lipoprotein Metabolism and Insulin Signaling. J Diabetes Res, 2017; 2017: 8356537 
40) Aung LH, Yin RX, Wu JZ, Wu DF, Wang W and Li H: Association between the MLX interacting protein-like, BUD13 homolog and zinc finger protein 259 gene polymorphisms and serum lipid levels. Sci Rep, 2014; 4: 5565

41) Zhang QH, Yin RX, Chen WX, Cao XL and Chen YM: Association between the TIMD4-HAVCR1 variants and serum lipid levels, coronary heart disease and ischemic stroke risk and atorvastatin lipid-lowering efficacy. Biosci Rep, 2018; 38: BSR20171058

42) De Castro-Oros I, Civeira F, Pueyo MJ, Mateo-Gallego R, Bolado-Carrancio A, Lamiquiz-Moneo I, Alvarez-Sala L,
Fabiani F, Cofan M, Cenarro A, Rodriguez-Rey JC, Ros E and Pocovi M: Rare genetic variants with large effect on triglycerides in subjects with a clinical diagnosis of familial vs nonfamilial hypertriglyceridemia. J Clin Lipidol, 2016; 10: 790-797

43) Wang J, Cao H, Ban MR, Kennedy BA, Zhu S, Anand S, Yusuf S, Pollex RL and Hegele RA: Resequencing genomic DNA of patients with severe hypertriglyceridemia (MIM 144650). Arterioscler Thromb Vasc Biol, 2007; 27: 24502455 